\title{
A Controlled Clinical Trial of A Novel Antivenom in Patients Envenomed by Bungarus multicinctus
}

\author{
Ha Tran Hung • Jonas Höjer • Trinh Xuan Kiem • \\ Nguyen Thi Du
}

Published online: 1 April 2010

(C) American College of Medical Toxicology 2010

\begin{abstract}
In northern Vietnam, a majority of severely envenomed patients are bitten by Bungarus multicinctus. Hitherto, these victims have received supportive care only. The aims of this study were to assess the possible efficacy and side effects of a new antivenom. This trial (ClinicalTrials.gov Identifier: NCT00811239) was performed during 2004-2006 at an ICU in Hanoi. For ethical reasons, the study was not randomized. All patients who fulfilled the inclusion criteria during 2004-2005 were prospectively enrolled, carefully recorded, and treated with optimal supportive therapy (control group). The patients who entered the study 2006 were treated with antivenom in addition to supportive care (antivenom group). The inclusion criteria were: envenomation by $B$. multicinctus, presence of systemic envenomation, and (during 2006) provision of written informed consent. Predefined endpoints were number of patients requiring mechanical ventilation, duration of mechanical ventilation, length of ICU stay, duration of muscle paralysis, and number of patients with ventilatorassociated pneumonia. Eighty-one patients were included, 54 during 2004-2005 and 27 during 2006. Baseline characteristics were similar in the groups. The antivenomgroup patients had a shorter duration of muscle paralysis of the limbs $(p<0.001)$, of the diaphragm $(p<0.001)$, and of ptosis $(p<0.001)$. The duration of mechanical ventilation and
\end{abstract}

Previous presentation: no data of this manuscript has previously been presented.

H. T. Hung • T. X. Kiem • N. T. Du

Vietnam Poison Control Center, Hanoi Medical University,

Hanoi, Vietnam

J. Höjer $(\bowtie)$

Swedish Poisons Information Centre, Karolinska Institute,

17176 Stockholm, Sweden

e-mail: jonashojer@hotmail.com length of ICU stay were shorter in the antivenom group $(p<0.001)$. The rate of ventilator-associated pneumonia was lower in the antivenom group $(p<0.02)$. However, the relative number of patients requiring mechanical ventilation was not reduced in the antivenom group. The rate of adverse reactions to the antivenom was $7.4 \%$. A favorable efficacy and acceptable safety of this antivenom were demonstrated.

Keywords Antivenom $\cdot$ Snakebite $\cdot$ Bungarus multicinctus $\cdot$ Vietnam

\section{Introduction}

Venomous snakebites constitute a serious health problem in many Asian countries. It has been estimated that approximately 25,000-35,000 people die each year from snakebite in that part of the world [1]. In Vietnam, the burden of snakebites on public health motivated Calmette to conduct original studies at the Vaccine Institute in Saigon over a hundred years ago and to develop the first snake antivenom ever [2].

In northern Vietnam, a vast majority of the most severely envenomed patients are bitten by Bungarus multicinctus (many-banded krait, Chinese krait), which is the only krait species giving rise to significant morbidity and mortality in the area. Its venom contains toxins which can cause severe neuromuscular blockade, but which do not give rise to swelling or necrosis at the site of the bite [1]. Alphabungarotoxin is a postsynaptically active toxin which binds to the acetylcholine receptors preventing binding of acetylcholine. The result is a non-depolarizing type of neuromuscular blockade. Moreover, the venom also contains $\beta$ and $\gamma$-bungarotoxins which act presynaptically and depress the release of acetylcholine from the nerve endings. 
The presence of the latter toxins explains why attempts at treatment with cholinesterase inhibitors, such as neostigmine, have not been very effective $[3,4]$.

Supportive care is an important part of the management of snakebites, but antivenom administration is the mainstay therapy in the majority of medically significant cases of envenoming. Such specific therapy may dramatically reduce the consequences of the envenomation [5-7]. Although antivenoms against $B$. multicinctus are available in China and Taiwan, clinical reports regarding their efficacy have only rarely been published $[3,8,9]$. In some series of cases, antivenom treatment has been tried after snakebites by other Bungarus species, but with rather conflicting clinical results $[10,11]$. In Vietnam, no specific antivenom against $B$. multicinctus has been available until recently, when it was developed and produced for clinical use. The aims of this study were to assess the effectiveness of this newly produced antivenom and to investigate its possible side effects.

\section{Materials and Methods}

This prospective controlled clinical trial was carried out during a 3-year period (2004-2006) at the Toxicological Intensive Care Unit (ICU) of Bach Mai Hospital in Hanoi. For ethical reasons and because the antivenom was not clinically available until 2006, the study was not randomized or blinded. All patients who fulfilled the inclusion criteria during the first two years of the study were prospectively enrolled, carefully monitored, and treated with optimal supportive therapy in the ICU (control group). The patients who entered the study during the third year were treated with antivenom therapy in addition to supportive care (antivenom group). The patients were enrolled on the basis of the following criteria: (1) envenomation by B. multicinctus, (2) presence of clinical signs of systemic envenomation (neuromuscular signs), and (3) (during the year 2006) provision of written informed consent (by close relative if the patient was unable to do so). Clinical data including a number of defined clinical and laboratory examinations were recorded for each case in a predetermined standardized protocol during the entire 3-year study period. The severity of the symptoms on recruitment was defined on the basis of the five-graded $(0-4)$ Poisoning Severity Score (PSS) [12].

During the year 2006, the patients were informed about the possible benefits and risks of the antivenom treatment and were able to withdraw at any time during the trial for any reason. Five to ten ampoules of antivenom, depending on the severity of muscle paralysis, were diluted with isotonic glucose solution to a total of $50 \mathrm{ml}$ and then infused intravenously by electric pump for a period of $1 \mathrm{~h}$. The patients were monitored continuously during and after the infusion to assess the efficacy of the treatment and any adverse reactions. Six to $8 \mathrm{~h}$ after the end of the infusion, a second infusion was administered, under similar conditions, if no clinical improvement or adverse reaction had been noted. Clinical examinations, in accordance with the study protocol, were performed at least twice daily up to discharge. The patients were also followed up 1 month later and readmitted if associated symptoms developed or became worse.

Predefined outcome endpoints were number of patients requiring mechanical ventilation, duration of mechanical ventilation, length of stay in the ICU, duration of a defined degree of muscle paralysis, and number of patients who developed a ventilator-associated pneumonia. The study was conducted in accordance with the WHO Good Clinical Practice guidelines and the Declaration of Helsinki. The study design and the form for the written consent were approved by the Ethics Committee of Hanoi Medical University. Statistical analyses were performed using an independent-samples $t$ test or the Mann-Whitney test for continuous variables and the $\chi^{2}$ test or Fisher's exact test for categorical variables. Differences were considered significant if the $p$ value was $<0.05$.

\section{Antivenom}

Venom pools from more than 100 specimens of Bungarus candidus and B. multicinctus of different sizes, ages, and sexes were collected from Vietnam. After milking, venoms were immediately lyophilized and then detoxified with glutaraldehyde, filtered, and stored at $4^{\circ} \mathrm{C}$ in bottles. Immunization of horses with increasing doses of the antigen described above was performed once a month for 7 months. The immunoglobulin fraction from each of the pooled horse plasma samples was pepsin-digested and then salt-precipitated to produce $\mathrm{F}\left(\mathrm{ab}^{\prime}\right)_{2}$ fragments. The antivenom was supplied in liquid form, 2,000 units per ampoule. The antivenom was approved for the use under discussion in the manuscript by the National Institute for Control of Medico Biological Products, Ministry of Health in Vietnam, but it has not yet become a commercial product.

\section{Results}

During the 3-year study period, a total of 81 patients fulfilled the inclusion criteria and entered the study. Of these, 54 patients were included during the years 2004 and 2005 (control group) and 27 during 2006 (antivenom group). The baseline characteristics were similar in the two groups, and are presented in Table 1. Most patients were male and of a productive age. Children below the age of 15 years 
Table 1 Baseline characteristics in the two study groups

\begin{tabular}{|c|c|c|c|}
\hline Variables & Control group & Antivenom group & Significance \\
\hline Age (years) & $34 \pm 15$ & $36 \pm 17$ & NS \\
\hline \multicolumn{3}{|l|}{ Sex } & \multirow[t]{3}{*}{ NS } \\
\hline Male & $38(70 \%)$ & $18(67 \%)$ & \\
\hline Female & $16(30 \%)$ & $9(33 \%)$ & \\
\hline Time to development of first symptom (hours) & $3.1 \pm 2.7$ & $2.7 \pm 1.9$ & NS \\
\hline \multicolumn{3}{|l|}{ Duration from snakebite to admission (number of patients) } & \multirow[t]{3}{*}{ NS } \\
\hline Within $6 \mathrm{~h}$ & $13(24 \%)$ & $9(33 \%)$ & \\
\hline$>6 \mathrm{~h}$ & $41(76 \%)$ & $18(67 \%)$ & \\
\hline \multicolumn{3}{|l|}{ Site of bite } & \multirow[t]{4}{*}{ NS } \\
\hline Limb & $49(91 \%)$ & $25(93 \%)$ & \\
\hline Trunk & $3(6 \%)$ & $1(4 \%)$ & \\
\hline Unknown & $2(4 \%)$ & $1(4 \%)$ & \\
\hline \multicolumn{3}{|l|}{$\begin{array}{l}\text { Severity score on recruitment } \\
\text { Moderate (PSS 2) }\end{array}$} & \multirow[t]{3}{*}{ NS } \\
\hline Moderate (PSS 2) & $9(17 \%)$ & $4(15 \%)$ & \\
\hline Severe (PSS 3) & $45(83 \%)$ & $23(85 \%)$ & \\
\hline
\end{tabular}

NS not significant; PSS poisoning severity score [12]

represented $14 \%$ of the study population and elderly patients ( $>60$ years) accounted for $4 \%$. Most of the patients developed their first symptoms within approximately $3 \mathrm{~h}$ after the snakebite, and were admitted to the ICU later than $6 \mathrm{~h}$ after the bite. All included patients displayed moderate or severe envenomation on admission according to the PSS criteria, with similar severity in the two groups.

The time span between the snakebite and the start of antivenom therapy was $19 \pm 9 \mathrm{~h}$, ranging from 5 to $38 \mathrm{~h}$. Twenty patients were treated by antivenom within $24 \mathrm{~h}$ after the bite (only two patients within $6 \mathrm{~h}$ ). The average dose of administered antivenom was $8.1 \pm 3.2$ ampoules, ranging from 5 to 17 ampoules.

The patients in the antivenom group had a significantly shorter duration of paralysis of the extremities, the diaphragm, and of ptosis. Hyponatremia was a common finding in the study population. The proportion of patients with marked hyponatremia (serum sodium level of less than $130 \mathrm{mmol} / \mathrm{L}$ ) was somewhat lower in the antivenom group, and severe hyponatremia (sodium level $<120 \mathrm{mmol} / \mathrm{L}$ or symptomatic) was only observed in the control group (two patients). The rate of ventilator-associated pneumonia was significantly lower in the antivenom group (Table 2).
Table 3 presents the treatment measures in the two groups. There was no fatality. The relative numbers of patients requiring endotracheal intubation and mechanical ventilation were similar in the two groups. However, the duration of mechanical ventilation and endotracheal intubation and the length of the ICU stay were significantly shorter in the antivenom group. No treatment with any cholinesterase inhibitor was given during the 3-year study period.

In the seven patients treated with antivenom later than $24 \mathrm{~h}$ after the snakebite, the duration of mechanical ventilation and the length of the ICU stay were longer than those in the 20 patients who received antivenom within $24 \mathrm{~h}$ (Mann-Whitney test, $p$ values 0.07 and 0.01 , respectively).

No patient in the study developed severe acute anaphylaxis. The rate of adverse reactions was $7.4 \%(2 / 27)$. A moderate allergic reaction in the form of urticaria was noted in one patient. Liver transaminase levels were transiently increased in another patient, in whom the maximum values of aspartate transaminase (AST) and alanine transaminase (ALT) were observed on the fifth day (344 and $442 \mathrm{U} / \mathrm{L}$, respectively, or approximately ten times the upper reference levels). Serum sickness was not observed in the study.

Table 2 A comparison between the two groups regarding some clinical findings during the stay in the intensive care unit

\begin{tabular}{llll}
\hline Clinical findings & Control group & Antivenom group & Significance $(p)$ \\
\hline Duration of limb paralysis (days) & $7.5 \pm 6.9$ & $2.2 \pm 2.6$ & $<0.001$ \\
Duration of diaphragm palsy (days) & $7.0 \pm 7.0$ & $1.6 \pm 1.6$ & $<0.001$ \\
Duration of ptosis (days) & $6.3 \pm 4.7$ & $3.5 \pm 1.5$ & $<0.001$ \\
Hyponatremia with serum Na $<130$ mmol/L (number of patients) & $27(50 \%)$ & $9(33 \%)$ & 0.16 \\
Ventilator-associated pneumonia (number of patients) & $27(50 \%)$ & $6(22 \%)$ & 0.02 \\
\hline
\end{tabular}


Table 3 A comparison between the two groups regarding some treatment measures during the intensive care unit (ICU) stay

\begin{tabular}{|c|c|c|c|}
\hline Outcome measures & Control group & Antivenom group & Significance $(p)$ \\
\hline \multicolumn{3}{|l|}{ Duration of mechanical ventilation (days) } & \multirow[t]{3}{*}{$<0.001$} \\
\hline Mean $( \pm$ SD) & $8.6 \pm 8.1$ & $2.3 \pm 2.5$ & \\
\hline Range & $0-31$ & $0-12$ & \\
\hline \multicolumn{3}{|l|}{ Duration of intubation (days) } & \multirow[t]{3}{*}{$<0.001$} \\
\hline Mean $( \pm \mathrm{SD})$ & $10.1 \pm 9.3$ & $3.0 \pm 2.6$ & \\
\hline Range & $0-40$ & $0-12$ & \\
\hline \multicolumn{3}{|l|}{ Length of ICU stay (days) } & \multirow[t]{3}{*}{$<0.001$} \\
\hline Mean $( \pm \mathrm{SD})$ & $11.6 \pm 9.7$ & $6.1 \pm 3.2$ & \\
\hline Range & $1-46$ & $1-15$ & \\
\hline Number of patients requiring mechanical ventilation & $44(81.5 \%)$ & $23(85.2 \%)$ & NS \\
\hline
\end{tabular}

NS not significant

Among the patients in the control group, none developed any allergic reaction and none had significantly but transiently elevated liver enzymes.

\section{Discussion}

The major findings in this clinical trial were the favorable clinical efficacy of the tested antivenom and the lack of severe adverse reactions to this treatment. The significant results of the study were achieved despite the fact that $93 \%$ of the patients in the interventional group received the antivenom therapy more than $6 \mathrm{~h}$ after the snakebite.

Although an improvement of most tested symptoms was demonstrated in the antivenom group in the present study, the relative number of patients requiring mechanical ventilation was not reduced. The considerable delay before ICU admission is the likely explanation. The reason why some patients had to stay endotracheally intubated also after they had been weaned from the ventilator (Table 3) was that a palatal paralysis with inability to swallow sometimes persisted longer than the paralysis of the respiratory muscles.

A high rate of hyponatremia was observed in the study population as was also reported in a previous study on snakebite by this particular species [13].

The average dose of the antivenom used in the present study was similar to the dose regimen used in a study in China, and in accordance with the recommended doses of other antivenom products used against bites by this Bungarus species [9]. With the aim of protecting its stability, the antivenom was not diluted in large amounts of solution in our study, but nevertheless was infused slowly during a period of $1 \mathrm{~h}$. Since skin testing is today not only considered unnecessary but also misleading, and pretreatment with antihistamine, epinephrine, or corticosteroids is controversial, neither a skin test nor any pretreatment was performed in the present study [14-17].
The reported frequencies of adverse reactions after different types of antivenom therapies have varied considerably. Acute reactions in $23-56 \%$ of the patients and high frequencies of serum sickness (up to $75 \%$ ) have been recorded, but in other studies the rates of these complications have been rather low or approximately $10 \%[5,18-20]$. The main explanation for this discrepancy is that the older immunoglobulin-based antivenoms had several side effects, whereas more recent, purer equine antivenoms, containing $\mathrm{F}\left(\mathrm{ab}^{\prime}\right)_{2}$ or Fab fragments, produce few side reactions [21]. In our study, no severe reactions were recorded and a single moderate reaction was observed in a patient who developed urticaria. This was probably due to the high quality of the $\mathrm{F}\left(\mathrm{ab}^{\prime}\right)_{2}$ antivenom in combination with rational dosing and slow administration. The transiently increased level of transaminases observed in another patient may possibly have had another, unknown explanation.

In view of the method of production of this new antivenom, it seems reasonable to assume that it should also be effective after bites by B. candidus (Malayan krait). However, this remains to be shown in a clinical trial.

\section{Limitations of the Study}

Unfortunately, it was not possible to perform this study as a randomized placebo-controlled trial. Therefore, we cannot exclude that a certain degree of unintentional bias may have influenced the recordings in the study protocol, even though every effort to avoid this was undertaken.

\section{Conclusion}

In conclusion, the efficacy and safety of this new antivenom were clearly demonstrated in this controlled clinical trial of patients bitten by $B$. multicinctus. 
Acknowledgment The financial support for the project from Sida's Secretariat for Research Cooperation for the bilateral cooperation between Vietnam and Sweden is gratefully acknowledged.

\section{References}

1. Warrell DA (1995) Clinical toxicology of snakebite in Asia. In: Meier J, White J (eds) Handbook of clinical toxicology of animal venoms and poisons. CRC, Florida, pp 493-594

2. Cheng AC, Winkel KD (2001) Snakebite and antivenoms in the Asia-Pacific: wokabaut wantaim, raka hebou ("walking together"). MJA 175:648-651

3. Chan JC, Cockram CS, Buckley T, Young K, Kay R, Tomlinson B (1995) Envenoming by Bungarus multicinctus (many-banded krait) in Hong Kong. J Trop Med Hyg 98:457-460

4. Rowan E (2001) What does $\beta$-bungarotoxin do at the neuromuscular junction? Toxicon 39:107-118

5. Dart RC, McNally J (2001) Efficacy, safety, and use of snake antivenoms in the United States. Ann Emerg Med 37:181-188

6. White J (1998) Envenoming and antivenom use in Australia. Toxicon 36:1483-1492

7. Karlson-Stiber C, Salmonson H, Persson H (2006) A nationwide study of Vipera berus bites during one year-epidemiology and mortality of 231 cases. Clin Toxicol 44:25-30

8. Lalloo DG, Theakston RDG (2003) Antivenom tables. J Toxicol Clin Toxicol 41:317-327

9. Sawai Y, Kawamura Y, Toriba M, Kobayashi T, Wang NP, Li CB et al (1992) An epidemiological study on the snakebites in Guangxi Zhuang autonomous region, China in 1990. The Snake 24:1-15

10. Warrel DA, Looareesuwan S, White NJ, Theakston RD, Warrel MJ, Kosakarn W et al (1983) Severe neurotoxic envenoming by the Malayan krait Bungarus candidus (Linnaeus): response to antivenom and anticholinesterase. BMJ 286:678-680
11. Theakson DG, Phillips RE, Warrel DA, Galagedera Y, Abeysekera DT, Dissanayaka P et al (1990) Envenoming by the common krait (Bungarus caeruleus) and Sri Lanka cobra (Najar najar najar): efficacy and complications of therapy by Haffkine antivenom. Trans R Soc Trop Med Hyg 84:301-308

12. Persson H, Sjöberg GK, Haines JA, Pronczuk de Garbino J (1998) Poisoning severity score. Grading of acute poisoning. J Toxicol Clin Toxicol 36:205-213

13. Hung HT, Höjer J, Du NT (2009) Clinical features of 60 consecutive ICU-treated patients envenomed by Bungarus multicinctus. Southeast Asia J Trop Med Public Health 40:518-524

14. Heard K, O'Malley GF, Dart RC (1999) Antivenom therapy in the Americas. Drugs 58:5-15

15. Malasit P, Warrel DA, Chanthavanich P, Viravan C, Mongkolsapaya J, Singhthong B et al (1986) Prediction, prevention, and mechanism of early (anaphylactic) antivenom reaction in victims of snake bites. BMJ 292:17-20

16. Weisman RS, Lizarralde SS, Thompson V (1996) Snake and spider antivenin: risks and benefits of therapy. J Florida M A 83:192-195

17. Lalloo DG, Theakston RDG (2003) Snake antivenoms. J Toxicol Clin Toxicol 41:277-290

18. Açikalin A, Gökel Y, Kuvandik G, Duru M, Köseoglu Z, Satar S (2008) The efficacy of low-dose antivenom therapy on morbidity and mortality in snakebite cases. Am J Emerg Med 26:402-407

19. Tariang DD, Philip PJ, Alexander G, Macaden S, Jeyaseelan L, Peter JV et al (1999) Randomized controlled trial on the effective dose of anti-snake venom in cases of snake bite with systematic envenomation. JAPI 47:369-371

20. Chippaux JP, Lang J, Eddine SA, Fagot P, Rage V, Peyrieux JC et al (1998) Clinical safety of a polyvalent $\mathrm{F}(\mathrm{ab})_{2}$ equine antivenom in 223 African snake envenomations: a field trial in Cameroon. Trans R Soc Trop Med Hyg 92:657-662

21. Karlson-Stiber C, Persson H, Heath A, Smith D, Al-Abdulla IH, Sjöström L (1997) First clinical experience with specific sheep Fab fragments in snake bite. Report of a multicentre study of Vipera berus envenoming. JIM 241:53-58 